\title{
Temperature Variations in PN Hydro Models
}

\section{Garrelt Mellema}

Stockholm Observatory

The are often inconsistencies between the results of different methods for deriving electron temperatures from line strengths in PNe. Temperatures derived from forbidden lines ratios such as [OIII]4363/5007, are on average higher than those calculated from permitted lines. This is normally explained by assuming the existence of areas of higher temperatures which have more weight in a forbidden line analysis, the so-called 'temperature fluctuations'. The magnitude of these fluctuations is expressed by the $t^{2}$ parameter. See Peimbert (1967). A typical value is about 0.03 , corresponding to fluctuations of $\sim 10 \%$. Photoionization models have difficulty in explaining fluctuations of this magnitude (Kingdon \& Ferland 1995).

I looked for temperature fluctuations in the output of a numerical hydrodynamic simulation for the formation of a PN. This is interesting because the hydrodynamic model includes the effects of shocks. The formation of the PN is modelled using the Interacting Stellar Winds model for aspherical PNe. In this model the nebula is shocked AGB wind material, and the shock velocity and strength increase from equator to pole due to a nonuniform density in the AGB-wind. See e.g. Mellema \& Frank (1995). In this simulation the density varies by a factor of 10 from equator to pole. The equatorial AGB mass loss rate is $510^{-5} M_{\odot} \mathrm{yr}^{-1}$ with a velocity of $20 \mathrm{~km} \mathrm{~s}^{-1}$. The fast wind has $510^{-8} M_{\odot} \mathrm{yr}^{-1}$ and $2000 \mathrm{~km} \mathrm{~s}^{-1}$. The shape of the nebula is roughly rectangular (similar to for example IC 4406).

The results of the simulation show strong temperature variations in the PN material. The dense gas near the equator has has a temperature around $9000 \mathrm{~K}$, whereas the gas near the pole has temperatures between 20,000 and $50,000 \mathrm{~K}$. The reasons for this are two. At the poles the nebula has a higher shock velocity $\left(73 \mathrm{~km} \mathrm{~s}^{-1}\right.$ against $\left.28 \mathrm{~km} \mathrm{~s}^{-1}\right)$, leading to more heating, and a lower density $\left(10^{3}-310^{3}\right.$ against $\left.1.210^{4} \mathrm{~cm}^{-3}\right)$, leading to less efficient cooling.

By calculating [OIII] and mean electron temperatures from the output of the simulation, I derived values for $t^{2}$. This parameter is high near the poles, up to 0.8 . But if one takes averages over strips along the major axis of the nebula (approximating long slit observations), the derived value for $t^{2}$ is at most 0.024 , a little less than typically observed. The reason for the difference is that the regions with a higher $T_{\mathrm{e}}$ and $t^{2}$ are also much fainter, and do not influence the average much.

One should be able to find these high temperature regions observationally through either position dependent line strength analysis or ratios of narrowband images. The results to date do not show any variations but they may not be sensitive enough. The high temperature regions in the simulation are at least 100 times fainter than the brightest parts of the nebula.

\section{REFERENCES}

Kingdon, J.B., Ferland, G.J., 1995, ApJ 450, 691

Mellema, G., Frank, A., 1995, Annals of the Israel Phys. Soc. 11, 229

Peimbert, M., 1967, ApJ 150, 825 